\title{
Expression of a Metalloproteinase That Degrades Native Type V Collagen and Denatured Collagens by Cultured Human Alveolar Macrophages
}

\author{
Margaret S. Hibbs, John R. Hoidal, and Andrew H. Kang \\ Department of Medicine, University of Tennessee at Memphis and Veterans Administration Medical Center, Memphis, Tennessee 38104
}

\begin{abstract}
Human pulmonary alveolar macrophages obtained by bronchoalveolar lavage from both normal controls and smokers secreted in vitro a neutral proteinase that degraded denatured collagens. Optimal expression of the proteinase was detected after 3-5 d of culture. The proteinase could not be detected in the media of cultures that had been treated with $0.5 \mu \mathrm{g} / \mathrm{ml}$ of cycloheximide. The gelatinase had an $M_{\mathrm{r}}$ of 90,000 and was immunologically cross-reactive with human neutrophil gelatinase. When newly synthesized ${ }^{35} S$-methionine-labeled proteins were analyzed, the proteinase appeared to be a major secretion product of alveolar macrophages. Chromatography on gelatin-Sepharose gave a single peak of activity that was predominantly composed of the 90,000 -mol-wt proteinase. The proteolytic activity in the gelatin-Sepharose-purified material was inhibited by EDTA and 1,10-phenanthroline, but not by $\boldsymbol{N}$-ethylmaleimide or phenylmethanesulfonyl fluoride, indicating that the proteinase was a metalloproteinase. The partially purified material was also capable of degrading native type $V$ collagen and this degradation was inhibited in the presence of an antibody to neutrophil gelatinase. The data suggest that human alveolar macrophages in culture elaborate a metalloproteinase that degrades both native type $V$ collagen and denatured collagens.
\end{abstract}

\section{Introduction}

The major human phagocytes, the monocyte-macrophage and the neutrophil, are derived from a common precursor in the bone marrow. However, many of their characteristics during maturation in the bone marrow and after release into the circulation are divergent. Neutrophils differentiate within the bone marrow during which time they synthesize most, if not all, their proteinases destined for later intracellular digestion or extracellular release (1). Upon release from the bone marrow, the neutrophil has a short life span during which its proteinases are either released in response to specific stimuli $(2,3)$ or nonspecifically released upon cell death. In contrast, cells of the monocyte lineage are long-lived, versatile cells capable of adopting multiple phenotypes in response to developmental and environmental regulators (4).

Although resident macrophages may be relatively quiescent in normal tissue, they show a striking increase in their

Address reprint requests to Dr. M. S. Hibbs, Research Service (151), Veterans Administration Medical Center, 555 Willard Ave., Newington, CT 06111.

Received for publication 17 September 1986 and in revised form 14 May 1987.

The Journal of Clinical Investigation, Inc.

Volume 80, December 1987, 1644-1650 secretory repetoire during inflammatory processes. Among their secretory products, in such a circumstance, are neutral proteinases, including metalloproteinases such as interstitial collagenase and elastase $(5,6)$. Thus, in an inflammatory state, the expression of neutral proteinases involved in connective tissue destruction appears to be enhanced. Such proteinases may directly contribute to the tissue destruction seen in diseases such as rheumatoid arthritis and pulmonary emphysema.

Over the past decade, our knowledge of the collagenous composition of the extracellular matrix has greatly expanded. At present the collagen family is comprised of $>25$ distinct gene products that encode at least 11 collagen types $(7,8)$. Corresponding studies of the proteinases related to collagenous matrix turnover were given impetus by the observation that traditional interstitial collagenases were incapable of degrading types IV and V collagens $(9,10)$. Subsequently, a number of proteinases have been described that degrade the various minor collagenous components of the extracellular matrix.

Among these proteinases is a metalloproteinase that has been termed by various investigators as gelatinase (11-13), type V collagenase (14), or metalloproteinase II (15). This proteinase degrades native type $\mathrm{V}$ collagen and its cartilage counterpart, type XI collagen. Whereas it also degrades denatured collagens of all types, it shows no activity against native types I, II, III, and IV collagens. The proteinase derived from neutrophils appears to be relatively specific for collagenous components of the extracellular matrix as it does not catalyze the degradation of the glycoproteins present in the matrix (12). Similar proteinases have been described in rabbit bone cultures (16) and "activated" rabbit alveolar macrophage cultures (14). As types V and XI collagens are pericellular collagens that are important in cell matrix communication (17), their depletion may lead to abnormal repair processes after inflammatory events.

In this study, we have examined the ability of human alveolar macrophages to secrete a metalloproteinase that degrades native type $\mathrm{V}$ collagen as well as denatured collagens. The identification of a gelatinase with functional and immunological similarities to neutrophil gelatinase suggests that human macrophages may play a direct role in the observed connective tissue remodeling that accompanies inflammation.

\section{Methods}

Cell preparation and culture. Human alveolar macrophages were obtained by bronchoalveolar lavage from ten cigarette smokers undergoing routine diagnostic procedures or two nonsmoking, drug-free normal volunteers. All cell populations utilized contained $>85 \%$ alveolar macrophages. Contamination by polymorphonuclear leukocytes was always $<5 \%$. The remaining cells identified were lymphocytes. The cell composition was determined by differential counts of Wright-Giemsa stained material. The cells were cultured at a density of $10^{6}$ alveolar macrophages/well in 24-well plates (surface area $2.1 \mathrm{~cm}^{2}$, Linbro, Flow Laboratories, Inc., McClean, VA) in Dulbecco's modified Essential 
medium (Gibco, Grand Island, NY) containing $0.2 \%$ lactalbumin hydroylsate, $100 \mathrm{U} / \mathrm{ml}$ penicillin, $100 \mu \mathrm{g} / \mathrm{ml}$ streptomycin, and 0.25 $\mu \mathrm{g} / \mathrm{ml}$ amphotericin B. $2 \mathrm{~h}$ after plating, the nonadherent cells were removed by washing three times with culture media. The adherent population was $>95 \%$ macrophages, as determined by nonspecific esterase staining (18). The culture media was again changed $24 \mathrm{~h}$ after plating and then at 2-3 d intervals. The aspirated media were treated with Tris- $\mathrm{HCl}, \mathrm{pH} 7.6$ (final concentration, $20 \mathrm{mM}$ ), phenylmethanesulfonyl fluoride (PMSF; final concentration, $2 \mathrm{mM}$ ), ${ }^{1}$ and $\mathrm{NaN}_{3}$ (final concentration, $0.02 \%$ ) and then frozen at $-20^{\circ} \mathrm{C}$ until analysis.

Collagen preparation. Type I collagen was prepared from an acid extract of fetal bovine skin by the method of Glimcher et al. (19) and radiolabeled as described by Cawston and Barrett (20). Type V collagen was isolated from human amnion by the procedure of Rhodes and Miller (21) and was a gift from Dr. Jerome M. Seyer.

Assay methods. Gelatinolytic activity was determined by the method of Harris and Krane (22) using heat-denatured (15 min at $60^{\circ} \mathrm{C}$ ) bovine type I collagen as the substrate, as previously described (13). Units of enzyme activity are expressed in terms of micrograms of gelatin degraded per min. Type $\mathbf{V}$ collagenolytic activity was determined by incubating $100 \mu \mathrm{g}$ of native type $\mathrm{V}$ collagen with the enzyme preparations in a reaction mixture containing $60 \mathrm{mM}$ Tris- $\mathrm{HCl}, 5 \mathrm{mM}$ $\mathrm{CaCl}_{2}, 0.1 \%$ Triton X-100, $0.02 \% \mathrm{NaN}_{3}, \mathrm{pH} 7.6$, for $18 \mathrm{~h}$ at $32.5^{\circ} \mathrm{C}$. After termination of the assay by the addition of EDTA (final concentration, $10 \mathrm{mM}$ ), the reaction products were analyzed on $7.5 \%$ polyacrylamide gels. Previous viscometric studies have indicated that the incubation temperature was below the melting temperature of type $\mathrm{V}$ collagen (14). All assays utilizing unpurified material were done in the presence of $2 \mathrm{mM}$ PMSF. To activate any latent enzyme present aminophenylmecuric acetate was added to the incubation mixture at a final concentration of $1 \mathrm{mM}(23)$.

Demonstration of enzyme activity in SDS-polyacrylamide gels. When gelatinase activity was to be determined, conventional SDS-gels (24) were run in the usual manner except that gelatin $(2 \mathrm{mg} / \mathrm{ml})$ was included in the running gel, the samples were not boiled or reduced before electrophoresis and the gel was cooled to $4^{\circ} \mathrm{C}$ during electrophoresis. After electrophoresis the gel was washed in $2.5 \%$ Triton X-100 $(\mathrm{wt} / \mathrm{vol})$ to renature the proteinase, rinsed in water, and then incubated in $50 \mathrm{mM}$ Tris- $\mathrm{HCl}, 5 \mathrm{mM} \mathrm{CaCl}_{2}, 0.001 \mathrm{mM} \mathrm{ZnCl}_{2}, 0.02 \% \mathrm{NaN}_{3}, \mathrm{pH}$ 7.6 , for $16 \mathrm{~h}$ at $37^{\circ} \mathrm{C}(25)$, as previously described (13). The zones of enzyme activity were indicated by negative staining.

Immunoblotting. Samples for immunoblotting were concentrated tenfold by pressure dialysis using a YM-10 membrane (Amicon Corp., Danvers, MA). The samples were electrophoresed on SDS-polyacrylamide gels and then transferred electrophoretically to nitrocellulose by the method of Towbin et al. (26) and developed as previously described (13).

Gelatin-Sepharose chromatography. Gelatin-Sepharose was prepared by linking heat-denatured bovine type I collagen to $\mathrm{CNBr}$-activated Sepharose (reference 13; Pharmacia Fine Chemicals, Piscataway, $\mathrm{NJ})$. Pooled supernatants from alveolar macrophage cultures were concentrated tenfold by pressure dialysis, dialyzed against the starting buffer, and applied to a $1 \times 10 \mathrm{~cm}$ column that had been previously equilibrated with $20 \mathrm{mM}$ Tris- $\mathrm{HCl}, 5 \mathrm{mM} \mathrm{CaCl}_{2}, 0.05 \%$ Brij-35 (vol/ vol), $0.02 \% \mathrm{NaN}_{3}$ (wt/vol), $\mathrm{pH} 7.6$, containing $150 \mathrm{mM} \mathrm{NaCl}$ (starting buffer). Flow rate was $40 \mathrm{ml} / \mathrm{h}$ and $5 \mathrm{ml}$ fractions were collected. The column was washed with the starting buffer until the absorbance had returned to baseline and then the column was washed with the starting buffer containing $1 \mathrm{M} \mathrm{NaCl}$. The proteolytic activity was eluted with 1 $\mathrm{M} \mathrm{NaCl}$ and 5\% dimethyl sulfoxide ( $\left.\mathrm{Me}_{2} \mathrm{SO}\right)$ in the starting buffer.

Antibody preparation. Antibodies were prepared, as previously described (13), either by immunizing with the $92-\mathrm{kD}$ form of neutrophil gelatinase obtained by preparative gel electrophoresis or by immunizing with the purified native form of the proteinase. Both antibodies

1. Abbreviations used in this paper: $\mathrm{Me}_{2} \mathrm{O}$, dimethyl sulfoxide; $\mathrm{PMSF}$, phenylmethanesulfonyl fluoride. reacted with all three forms of neutrophil gelatinase and were shown to be monospecific by immunoblotting techniques. However, the antibody against the native form of the proteinase was more effective in immunoprecipitation studies. Purified IgG was obtained by affinity chromatography on protein A Sepharose (27).

${ }^{35} S$-methionine labeling and immunoprecipitation studies. Alveolar macrophages that had been cultured for $48 \mathrm{~h}$ were washed three times with methionine-free DME and then incubated with methionine-free DME supplemented with $1 \mathrm{mM}$ methionine and $50 \mu \mathrm{Ci}{ }^{35} S$-methionine (New England Nuclear, Boston, MA) for $16 \mathrm{~h}$. The labeled media proteins were precipitated by quinine sulfate (28). Immunoprecipitation was performed, as described by Mischel et al. (29), using $10 \mu \mathrm{g}$ of purified IgG of either preimmune or immune serum except that Empigen was substituted for NP-40 as the detergent. Precipitated proteins were analyzed on $10 \%$ polyacrylamide gels. After fixation and treatment with Enhance (New England Nuclear) the gels were dried and fluorographed as previously described (30). Densitometry was performed using a laser densitometer (LKB Instruments, Inc., Gaithersburg, MD) equipped with an integrator (Hewlett-Packard, Co., Palo Alto, CA).

\section{Results}

Identification of gelatinolytic activity in the media of alveolar macrophage cultures. Initially, the culture supernatants were assayed for functional gelatinase activity as described in the Methods. Alveolar macrophages from both normal volunteers and smokers secreted gelatinolytic activity into the culture media (Fig. 1). While the maximal enzyme activity was noted in the 1st 3-5 d of culture, continued production of enzyme activity was noted for up to $12 \mathrm{~d}$ of culture. The enzymatic activity was present in a latent form ( $>99 \%)$. The functional activity from the various cell preparations differed substantially with the total cumulative activity ranging from 8 to 26 $\mathrm{U} / 10^{7}$ cells. The basis for the variability in functional activity in these preparations is not clear; however, it is possible that the concomitant secretion of collagenase inhibitor in some cell preparations (31) may be responsible for the variability in functional gelatinase expression. Collagenase inhibitor is also an effective gelatinase inhibitor (Hibbs M. S., and G. P. Stricklin, unpublished observations). As similar results were obtained with cells from smokers and nonsmokers, the remainder of the studies were performed with cells from smokers since the number of cells obtained were higher.

The spectrum of gelatinolytic proteinases present in the macrophage culture media was similar in all preparations as determined by a substrate gel technique. The majority of the gelatinolytic activity was detected at an $M_{\mathrm{r}}$ of 90,000 (Fig. 2). Some higher molecular weight material $(250,000 \mathrm{D})$ was also noted. It is unclear at present whether the higher molecular

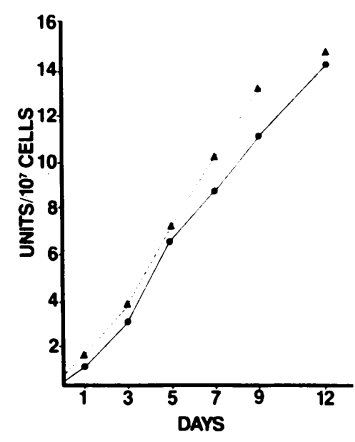

Figure 1. Cumulative gelatinase activity in alveolar macrophage culture media. Alveolar macrophages from normal individuals or uninfected smokers were obtained by bronchoalveolar lavage and cultured. Functional gelatinase activity in the media was determined using denatured ${ }^{14} \mathrm{C}$-labeled type I collagen. Results are expressed as $U / 10^{7}$ macrophages. A representative experiment is shown.

$-\bullet-$, Smoker; $-\wedge-$, nonsmoker. 


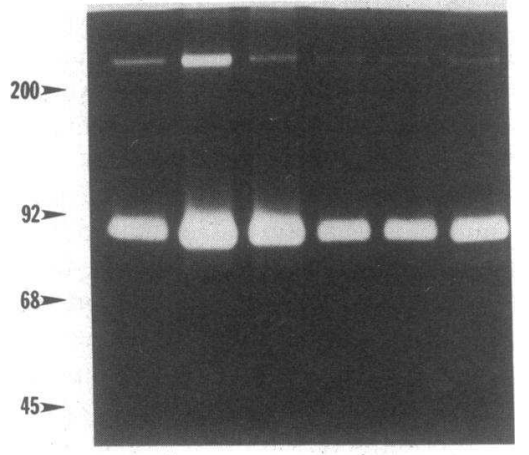

$13 \underset{\text { DAYS }}{5} \quad 7 \quad 9 \quad 12$
Figure 2. Identification of gelatinase by substrate gel analysis. $60 \mu \mathrm{l}$ of concentrated (tenfold) conditioned media were electrophoresed on an SDS-polyacrylamide gel $(6 \%)$ containing 2 $\mathrm{mg} / \mathrm{ml}$ gelatin. After renaturation of the enzyme by washing the gel in $2.5 \%$ Triton, the gels were incubated for $16 \mathrm{~h}$ at $37^{\circ} \mathrm{C}$. Zones of negative staining represent areas of enzyme activity.

weight form represents the native secreted species of the enzyme or an aggregate. Such large molecular weight species have also been observed in our studies of neutrophil gelatinase (13). Further biosynthetic studies will be necessary to resolve these questions.

Immunoreactivity of the macrophage proteinase with neutrophil gelatinase. As the major molecular weight species seen on gelatin-substrate gels was similar to the molecular weight of one of the forms of neutrophil gelatinase (13), the reactivity of this protein with a polyclonal antibody prepared against the 92-kD species of neutrophil gelatinase was examined by immunoblotting. Strong reactivity at $M_{\mathrm{r}}$ of 90,000 was noted in the media from the 3-5-d cultures (Fig. 3) that corresponded to the peak activity seen on gelatin-substrate gels. Less reactivity was noted at other time periods, which may be related to the smaller amounts of antigen present. Although the immunoreactivity generally paralleled the activity as demonstrated by substrate gel analysis, it is possible that the neutrophil and macrophage proteinases share common antigenic determinants but are not completely immunologically identical.

Biosynthesis of gelatinase by alveolar macrophages. As several reports have indicated that alveolar macrophages secrete stored neutrophil proteinases $(32,33)$, studies were undertaken to establish that the detected gelatinolytic proteinase was a biosynthetic product of the macrophage. Initially, we examined the distribution of the proteinase between the culture media and the cell layer $48 \mathrm{~h}$ after the initial plating of the macrophages. This time point was chosen since any contami-

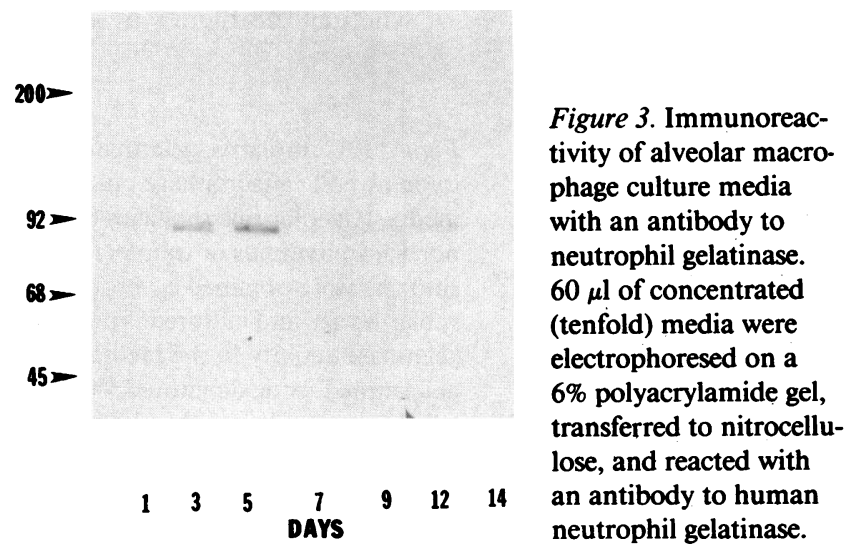

nating neutrophils should have died and released their proteinases for macrophage uptake (1). When the culture media and cell layer lysate were examined for gelatinolytic activity on substrate gels, activity was only detected in the culture media (Fig. $4 \mathrm{~A}$ ). These findings suggest that alveolar macrophages synthesize and secrete, rather than store, the gelatinolytic activity expressed in the culture supernatants.

Subsequently the ability of an inhibitor of protein synthesis, cycloheximide, to block the secretion of gelatinolytic proteinases into the culture media was examined. Alveolar macrophage cultures were treated with the indicated amounts of cycloheximide after $3 \mathrm{~d}$ in culture, and the media was harvested at day 5 . The culture media was then analyzed by gelatin-subtrate gels and functional gelatinase assay. Gelatinolytic activity was significantly inhibited by cycloheximide at a concentration of $0.5 \mu \mathrm{g} / \mathrm{ml}$ cycloheximide (Fig. $4 B$ ). These results suggested that protein synthesis was required for gelatinase production and/or release in these cultures.

To conclusively demonstrate that the proteinase was a biosynthetic product of the macrophage, macrophage cultures were labeled with ${ }^{35} S$-methionine. Analysis of the labeled secreted proteins revealed a prominent band at $90 \mathrm{kD}$ (Fig. 5). Densitometric analysis suggested that this protein represents $\sim 10 \%$ of the secreted proteins of the alveolar macrophage. Immunoprecipitation of the labeled culture media with an antibody against neutrophil gelatinase demonstrated a single polypeptide that migrated as a $90-\mathrm{kD}$ band. These results demonstrate that $90 \mathrm{kD}$ gelatinase is one of the major biosynthetic products of cultured alveolar macrophages.
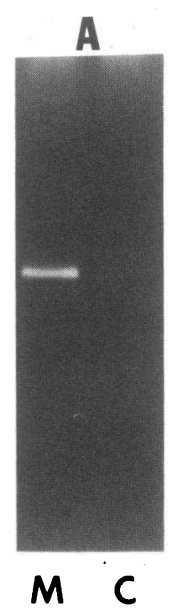

B

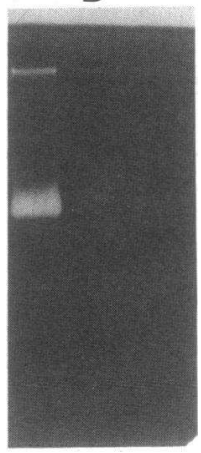

$0 \quad 0.55$ INHIBITION $\quad 96 \% \quad 95 \%$
Figure 4. Gelatinase expression is dependent on protein synthesis. $(A)$ The gelatinase in the culture media and cell layer extracts from $10^{7}$ macrophages was identified by substrate gel analysis. Media and cells were harvested after $48 \mathrm{~h}$ of culture. Media represents the amount of gelatinase secreted between 24 and $48 \mathrm{~h}$ in culture. (B) Alveolar macrophage cultures were treated with the indicated amount (micrograms per milliliter) of cycloheximide for $48 \mathrm{~h}$, and the culture media was analyzed for gelatinase activity by substrate gel analysis and functional gelatinase activity (percent inhibition). 


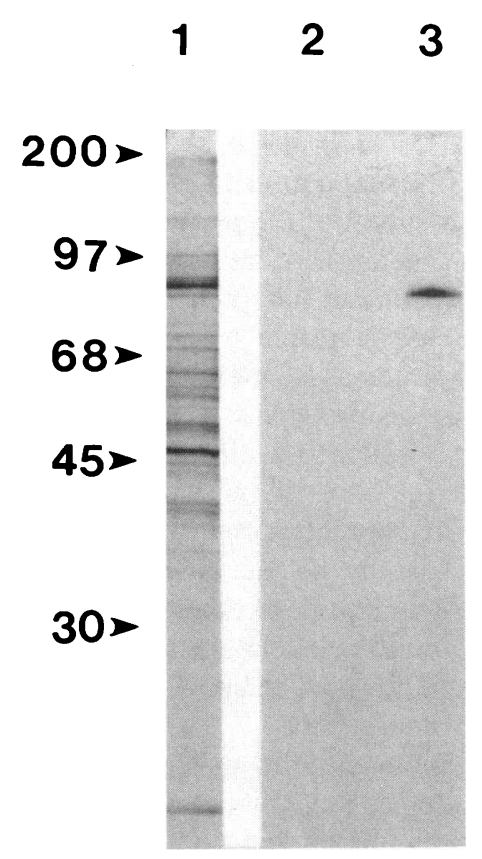

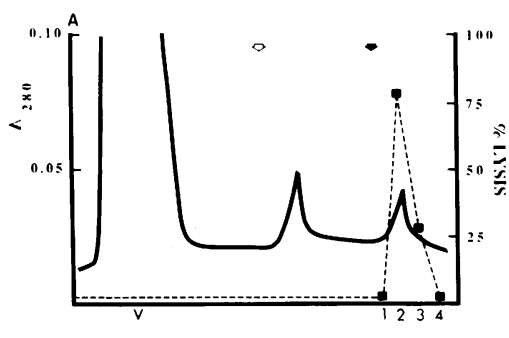

Figure 5. Immunoprecipitation of metabolically labeled macrophage gelatinase. Macrophages were incubated with ${ }^{35} S$-methionine for $16 \mathrm{~h}$ and the media processed as described in the Methods. Lane 1, Quinine sulfate precipitation of labeled proteins; lanes 2 and 3, immunoprecipitation; lane $2,10 \mu \mathrm{g}$ preimmune IgG; lane 3, 10 $\mu \mathrm{g}$ antineutrophil gelatinase IgG.
Characterization of the gelatinolytic activity present in the media of alveolar macrophage cultures. To facilitate characterization of the gelatinolytic proteinase secreted by alveolar macrophages, the proteinase was isolated from the culture media by affinity chromatography on gelatin-Sepharose as described in the Methods. Fig. $6 \mathrm{~A}$ shows that the gelatinolytic activity was sharply eluted with $\mathrm{Me}_{2} \mathrm{SO}$. When the fractions were screened on gelatin-substrate gels (Fig. $6 \mathrm{~B}$ ), the gelatinase activity present in the starting material was found to be totally adsorbed to the affinity matrix and was eluted in the $\mathrm{Me}_{2} \mathrm{SO}$ fraction. No activity was noted in the $1 \mathrm{M} \mathrm{NaCl}$ fraction (not shown). Although there were several minor bands of gelatinolytic activity in the concentrated material, the 90,000-mol-wt form is the major proteinase.

As we have observed the phenomenon of autodegradation in concentrated preparations of gelatinases purified from other sources (reference 13, unpublished observations), we examined the possibility that the additional lower molecular weight bands were related to autoactivation of the proteinase. The purified proteinase was incubated with $1 \mathrm{mM}$ aminophenylmercuric acetate at $37^{\circ} \mathrm{C}$ for $16 \mathrm{~h}$. Then the activated and the autoactivated material was analyzed by substrate gel analysis. As seen in Fig. 7, the molecular weight of the activated proteinase is clearly shifted to those corresponding to the minor bands of activity seen in the fraction obtained by gelatin-Sepharose chromatography. These data suggest that these minor bands of activity are related to autocatalysis.

After isolation of the proteinase, the inhibition of the gelatinase by standard proteinase inhibitors was examined. Metal chelating agents such as 1,10-phenanthroline and EDTA were effective inhibitors. The serine proteinase inhibitor, PMSF, and the thiol proteinase inhibitor, $\mathrm{N}$-ethylmaleimide, failed to inhibit the gelatinolytic activity (Table I). Also, if substrate gels of either the crude or the partially purified material were incubated in the presence of these proteinase inhibitors, only metal chelating agents were found to be effective inhibitors (not shown). These results demonstrated that the gelatinolytic activity was attributable to a metalloproteinase.

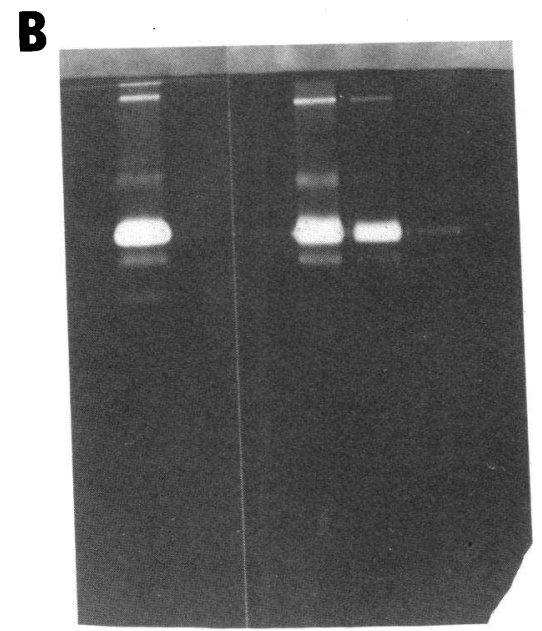

$S M V \quad 1 \quad 2 \quad 3 \quad 4$
Figure 6. Gelatin-Sepharose chromatography of alveolar macrophage culture media. $(A)$ Concentrated media that had been dialyzed against the starting buffer was applied to a $1 \times 10 \mathrm{~cm}$ column of gelatin-Sepharose equilibrated in $20 \mathrm{mM}$ Tris$\mathrm{HCl}, 5 \mathrm{mM} \mathrm{CaCl}$, 0.05\% Brij-35, 0.02\% $\mathrm{NaN}_{3}$ containing 150 $\mathrm{mM} \mathrm{NaCl}$ (starting buffer). After the absorbance had returned to baseline, the column was washed with the starting buffer containing $1 \mathrm{M} \mathrm{NaCl}$ (open arrow), and then the enzyme was eluted with the starting buffer containing $1 \mathrm{M} \mathrm{NaCl}$ and $5 \% \mathrm{Me}_{2} \mathrm{SO}$ (closed arrow). 1, 2, 3, and 4 are the fractions eluted with $1 \mathrm{M} \mathrm{NaCl} / 5 \%$

$\mathrm{Me}_{2} \mathrm{O}$ in the starting buffer and correspond to the fractions analyzed by substrate gel analysis. —-, Absorbance $(280 \mathrm{~nm}) ;---$, gelatinase activity; $\mathrm{V}$, void. $(B)$ The gelatinolytic activity of the fractions from the gelatin-Sepharose column as determined by substrate gel analysis ( $8 \%$ polyacrylamide). $1,2,3$, and 4 indicate the four fractions eluted with $\mathrm{Me}_{2} \mathrm{SO}$. No activity was seen in the $1 \mathrm{M} \mathrm{NaCl}$ fraction (not shown). SM, starting material; $\mathrm{V}$, void (unbound fractions pooled).

Since a subset of gelatinolytic proteinases have been shown to degrade type $V$ collagen (12-14), the ability of the gelatinase isolated by gelatin-Sepharose chromatography to degrade native type $\mathrm{V}$ collagen was examined. In these experiments, the proteinase was incubated with type $\mathrm{V}$ collagen for $18 \mathrm{~h}$ at $37^{\circ} \mathrm{C}$ and the reaction products were separated on a $6 \%$ polyacrylamide gel. As seen in Fig. 8, the material isolated by gelatinSepharose also degraded native type $\mathrm{V}$ collagen. To assure that this type $\mathrm{V}$ degrading activity was related to the $90,000-\mathrm{D}$ proteinase, the ability of the antibody to neutrophil gelatinase to inhibit the type $\mathrm{V}$ degradation was also assessed. When antineutrophil gelatinase IgG was included in the reaction mixture, the type $\mathrm{V}$ degradation was almost completely inhib-

A B

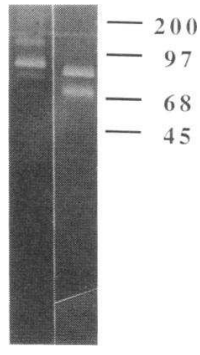

Figure 7. Effect of activation on the molecular weight of gelatinase. The proteinase fraction isolated by gelatin-Sepharose chromatography was incubated with $1 \mathrm{mM}$ aminophenylmecuric acetate for $16 \mathrm{~h}$ at $37^{\circ} \mathrm{C}$ and then analyzed on a $10 \%$ polyacrylamide substrate gel. Lane 1, unactivated proteinase; lane 2 , activated proteinase. 
Table I. Inhibition of Alveolar Macrophage Gelatinase*

\begin{tabular}{lll}
\hline Inhibitor & Activity & Inhibition \\
\hline & $\begin{array}{l}\mu g \text { gelatin degraded/min } \\
\text { per } m l\end{array}$ & $\%$ \\
& 3.7 & - \\
None & 0 & 100.0 \\
1,10-phenanthroline (5 mM) & 0.16 & 96.0 \\
EDTA (10 mM) & 3.5 & 5.0 \\
N-ethylmaleimide (10 mM) & 3.6 & 4.0 \\
PMSF (2 mM) & & \\
\hline
\end{tabular}

* $30 \mu \mathrm{l}$ of gelatin-Sepharose-isolated gelatinase were preactivated with $1 \mathrm{mM}$ aminophenylmercuric acetate for $1 \mathrm{~h}$ at $37^{\circ} \mathrm{C}$ and then incubated with $100 \mu \mathrm{g}$ gelatin for $6 \mathrm{~h}$ at $37^{\circ} \mathrm{C}$ in the presence of the indicated inhibitor.

ited (Fig. 8, lane 3). The inclusion of an irrelevant IgG had no effect on the degradation. These findings demonstrated that human alveolar macrophages elaborate a proteinase that degrades both denatured collagens and native type $\mathrm{V}$ collagen.

\section{Discussion}

In this report, we have identified a gelatinolytic proteinase that is secreted by cultured human alveolar macrophages. The proteolytic activity is present predominately in the form of a $90-\mathrm{kD}$ proteinase, though a higher molecular weight form is also evident. As has been reported for other neutral metalloproteinases secreted by monocyte-macrophages $(5,6)$, the proteinase does not appear to be stored in the cell and its secretion is dependent on active protein synthesis. Although cycloheximide could interfere with secretion in an indirect manner, the demonstration of immunoprecipitable, newly synthesized ${ }^{35} S$ labeled gelatinase clearly indicates that the proteinase is a biosynthetic product of alveolar macrophages. Also, this proteinase appears to be a major product of the macrophage.

Characterization of the proteolytic activity revealed that it appears to be due to a metalloproteinase. The partially purified material also exhibits the ability to degrade native type $\mathrm{V}$ collagen. Although the isolated material is predominantly $90 \mathrm{kD}$, a number of minor bands of gelatinolytic activity are also present. The higher molecular weight species could represent aggregates that form after concentration by the chromato-

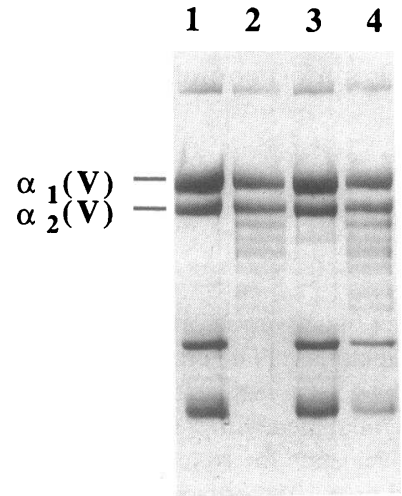

Figure 8. Degradation of type $\mathrm{V}$ collagen by gelatinase isolated by affinity chromatography and the inhibition of degradation in the presence of antineutrophil gelatinase IgG. $0.5 \mu \mathrm{g}$ of the gelatinase preparation obtained by gelatinSepharose chromatography were incubated with $50 \mu \mathrm{g}$ of type $\mathrm{V}$ collagen for $18 \mathrm{~h}$ at $32.5^{\circ} \mathrm{C}$ and the reaction products separated on a $7.5 \%$ polyacrylamide gel. Antibody dilution was 1:500. Lane 1 , collagen plus antigelatinase IgG; lane 2, collagen plus enzyme; lane 3 , collagen plus enzyme plus antigelatinase IgG; lane 4, collagen plus enzyme plus unrelated immune IgG. graphic procedure. Such species were also observed in our previous studies with neutrophil gelatinase. Those studies demonstrated that the higher molecular weight species seen in association with the $90-\mathrm{kD}$ proteinase also degraded type $\mathrm{V}$ collagen (13). The lower molecular weight bands appear to be related to autodegradation and partial activation of the proteinase during isolation. The spontaneous generation of multiple molecular weight species of metalloproteinases has been previously reported $(34,35)$. The ability of the neutrophil gelatinase antibody that recognizes the $90-\mathrm{kD}$ proteinase to inhibit the type $\mathrm{V}$ degrading activity indicates that this proteinase is responsible for both activities. Interestingly, similar to neutrophil gelatinase, the proteinase shows no detectable activity against native types I-IV collagens, elastin, or glycoproteins such as fibronectin or laminin (data not shown). Thus, the data are consistent with our previous studies $(13,14)$ and those of other investigators $(12,16)$ that have demonstrated that gelatin-specific metalloproteinases with a $M_{\mathrm{r}}$ in the range of 85,000-95,000 are consistently found to be type $\mathrm{V}$ collagenases.

Despite the complexities of the system, it is clear that human alveolar macrophages secrete proteolytic activity that degrades both denatured collagens and native type $\mathrm{V}$ collagen. Such proteolytic capacity may influence the extracellular matrix composition by several mechanisms. Gelatinases may facilitate the degradation of the interstitial collagens by its synergism with interstitial collagenase $(12,36)$. As type $\mathrm{V}$ collagen is felt to be important in cell matrix comunication (17), it is possible that degradation of this collagen may either lead to qualitative or quantitative alterations in the extracellular matrix and/or disorderly deposition of the newly synthesized interstitial collagens. However, further understanding of the biochemistry and cell biology of type $\mathrm{V}$ collagen will be required before the definitive determination of the pathophysiological effects of the depletion of this collagenous molecule from the extracellular matrix can be made.

Although collagenolytic metalloproteinases and their regulation have been relatively well studied in mononuclear phagocytes from mice and rabbits $(5,6,14,37)$, there remains a paucity of information about these proteinases in human monocyte-macrophages. Louie et al. (38) demonstrated interstitial collagenase activity in the media of peripheral blood monocyte cultures after $3 \mathrm{~d}$ of in vitro differentiation that could be upregulated by the addition of phorbol myristic acetate or concanavalin A. In contrast, Gadek et al. (39) reported that collagenase activity in cultures of human alveolar macrophages was expressed in a constitutive manner. The recent studies by Welgus et al. (31) using immunological assays have demonstrated that human alveolar macrophages secrete both collagenase and collagenase inhibitor. The regulation of collagenase and collagenase inhibitor was coordinate in response to lipopolysaccharide; however, when the cells were treated with phorbol diesters, only inhibitor levels increased. Human leukemic cell lines have also been shown to secrete interstitial collagenase and/or inhibitor after differentiation toward the macrophage phenotype $(40,41)$.

Interestingly, recent studies by Hasty et al. $(35,42,43)$ have definitively demonstrated that neutrophil collagenase is a distinct member of the interstitial collagenase family. Complimentary studies by Welgus and co-workers (31) have shown that the interstitial collagenase secreted by macrophages is immunologically and functionally identical to the fibroblast collagenase. In contrast, our data indicates that macrophage gela- 
tinase more closely resembles the neutrophil form of this proteinase (13) as the predominant gelatinase in fibroblast culture media is $67 \mathrm{kD}$ and the native form of fibroblast enzyme is not reactive with our antibodies to neutrophil gelatinase (44). Further studies will be needed to determine whether these differences are due to posttranslational processing or alterations in gene expression.

In addition to the studies on interstitial collagenase, a metal-dependent type IV collagenase has been detected in peripheral blood monocytes and certain types of macrophages in culture (45). In contrast to the interstitial collagenases, the expression of this proteinase was maximal within the $1 \mathrm{st} 6 \mathrm{~h}$ of culture and progressively declined after that time. Thus, it appears that human macrophages secrete several metalloproteinases that may participate in degradation of the collagenous components of the extracellular matrix. The detection of these proteinases may be impeded by the presence of collagenase inhibitor (tissue inhibitor of metalloproteinases) as well as by the complexities of their regulation.

In the present study, we have defined a third collagenolytic metalloproteinase, gelatinase-type $\mathrm{V}$ collagenase, as another secretory product of human macrophages. Its secretion generally paralleled the secretion of interstitial collagenase; however, gelatinase secretion appears to be more sustained. Although the data suggest that the proteinase is constitutively secreted, additional studies will be need to clearly define the regulation of this proteinase. Indeed, the further study of the collagenolytic metalloproteinases secreted by human macrophages in terms of both collagen type specificity and regulation of expression should provide important insights into the role of these cells in collagen degradation in both physiologic and pathologic processes.

\section{Acknowledgments}

We gratefully acknowledge the helpful comments of Drs. George P. Stricklin and Raj Rhagow.

This work was supported by U. S. Public Health Service grants AM01138, AM16506, and HL01308 and by funds from the Veterans Administration. Dr. Hibbs is a recipient of a Clinical Investigator Award from the National Institute of Arthritis, Musculoskeletal, and Skin Diseases. Dr. Hoidal is a recipient of a Research Career Development Award from the National Heart, Lung, and Blood Institute.

\section{References}

1. Bainton, D. F., J. L. Ullyot, and M. G. Farquar. 1971. Development of neutrophilic polymorphonuclear leukocytes in human bone marrow. J. Exp. Med. 134:907-934.

2. Weissman, G., J. E. Smolen, and H. M. Korchak. 1980. Release of inflammatory mediators from stimulated neutrophils. $N$. Engl. $J$. Med. 303:27-33.

3. Hibbs, M. S., K. A. Hasty, A. H. Kang, and C. L. Mainardi. 1984. Secretion of collagenolytic enzymes from human polymorphonuclear leukocytes. Collagen Relat. Res. 4:467-477.

4. Takemura, R., and Z. Werb. 1984. Secretory products of macrophages and their physiological functions. Am. J. Physiol. 246:C1-C9.

5. Werb, Z., and S. Gordon. 1975. Secretion of a specific collagenase by stimulated macrophages. J. Exp. Med. 142:346-360.

6. Werb, Z., and S. Gordon. 1975. Elastase secretion by stimulated macrophages. J. Exp. Med. 142:361-377.

7. Mayne, R. 1984. The different types of collagen and collagenous peptides. In The Role of the Extracellular Matrix in Development. R. L. Trelstead, editor. Alan R. Liss, Inc., New York. 33-42.
8. Miller, E. J. 1985. The structure of fibril forming collagens. Ann. NY Acad. Sci. 480:1-13.

9. Chiang, T. M., C. L. Mainardi, J. M. Seyer, and A. H. Kang. 1980. Collagen-platelet interaction: Type V (AB) collagen induces platelet aggregation. J. Lab. Clin. Med. 95:99-107.

10. Welgus, H. G., J. J. Jeffrey, and A. Z. Eisen. 1981. The collagen substrate specificity of human skin collagenase. J. Biol. Chem. 256:9511-9515.

11. Murphy, G., U. Bretz, M. Baggiolini, and J. J. Reynolds. 1980. The latent collagenase and gelatinase of human polymorphonuclear leucocytes. Biochem. J. 192:517-525.

12. Murphy, G., J. J. Reynolds, U. Bretz, and M. Baggiolini. 1982. Partial purification of collagenase and gelatinase from human polymorphonuclear leukocytes. Biochem. J. 203:209-221.

13. Hibbs, M. S., K. A. Hasty, J. M. Seyer, A. H. Kang, and C. L. Mainardi. 1985. Biochemical and immunological characterization of the secreted forms of human neutrophil gelatinase. J. Biol. Chem. 260:2493-2500.

14. Mainardi, C. L., M. S. Hibbs, K. A. Hasty, and J. M. Seyer. 1984. Purification of a type $\mathrm{V}$ collagen degrading metalloproteinase from rabbit alveolar macrophages. Collagen Relat. Res. 4:479-492.

15. Okada, Y., H. Nagase, and E. D. Harris, Jr. 1986. A multisubstrate metalloproteinase from rheumatoid synovial cells. Clin. Res. 34:732. (Abstr.)

16. Murphy, G., T. E. Cawston, W. A. Galloway, M. J. Barnes, R. A. D. Bunning, E. Mercer, J. J. Reynolds, and R. E. Burgeson. 1981. Metalloproteinases from rabbit bone culture medium degrade types IV and V collagens, laminin and fibronectin. Biochem. J. 199:807-811.

17. Gay, S., R. K. Rhodes, R. E. Gay, and E. J. Miller. Collagen molecules comprised alpha 1 chains (B chains): an apparent localization in the exocytoskeleton. Collagen. Relat. Res. 1:53-58.

18. Yam, L. T., C. Y. Li, and W. H. Crosby. 1971. Cytochemical identification of monocytes and granulocytes. Am. J. Clin. Pathol. 55:283-290.

19. Glimcher, M. J., C. T. Francois, L. Richards, and S. Krane. 1964. The presence of organic phosphorus in collagens and gelatins. Biochim. Biophys. Acta. 93:585-602.

20. Rhodes, R. K., and E. J. Miller. 1978. Physical characterization and molecular organization of collagen A and B chains. Biochemistry. 17:3442-3448.

21. Cawston, T. E., and A. Barrett. 1979. A rapid and reproducible assay for collagenase using $\left[1-{ }^{14} \mathrm{C}\right]$-acetylated collagen. Anal. Biochem. 99:340-345.

22. Harris, E. D., Jr., and S. M. Krane. 1972. An endopeptidase from rheumatoid synovial tissue culture. Biochim. Biophys. Acta. 258:4662-4668.

23. Sellers, A., E. Cartwright, G. Murphy, and J. J. Reynolds. 1977. Evidence that latent collagenases are enzyme-inhibitor complexes. Biochem. J. 163:303-307.

24. Laemmli, U. K. 1970. Cleavage of structural proteins during the assembly of the head of bacteriophage T4. Nature (Lond.). 227:680-685.

25. Birkedal-Hansen, H., and R. E. Taylor. 1982. Detergent activation of latent collagenase and resolution of its component molecules. Biochem. Biophys. Res. Commun. 107:1173-1178.

26. Towbin, H., T. Staehlin, and J. Gordon. 1979. Electrophoretic transfer of proteins from polyacrylamide gels to nitrocellulose sheets: procedure and some applications. Proc. Natl. Acad. Sci. USA. 76:4350-4354.

27. Oi, V. T., and L. A. Herzenberg. 1980. Immunoglobin-producing Hybrid cell lines. In Selected Methods in Cellular Immunology. B. B. Mischell and S. M. Shiigi, editors. W. H. Freeman and Co., San Francisco. 368-370.

28. Herron, G. S., Z. Werb, K. Dwyer, and M. J. Banda. 1986. Secretion of metalloproteinases by stimulated capillary endothelial cells: production of procollagenase and prostomelysin exceeds expression of proteolytic activity. J. Biol. Chem. 261:2810-2813.

29. Jones, P. P. 1980. Immunoprecipitation of cell proteins. In 
Selected Methods in Cellular Immunology. B. B. Mischell and S. M. Shiigi, editors. W. H. Freeman and Co., San Francisco. 407-411.

30. Hibbs, M. S., A. E. Postlethwaite, C. L. Mainardi, J. M. Seyer, and A. H. Kang. 1983. Alterations in collagen production in mixed mononuclear leukocyte-fibroblast cultures. J. Exp. Med. 157:47-59.

31. Welgus, H. G., E. J. Campbell, Z. Bar-Shavit, R. M. Senior, and S. L. Teitelbaum. 1985. Human alveolar macrophages produce a fibroblast-like collagenase and collagenase inhibitor. J. Clin. Invest. 76:219-224.

32. Campbell, E. J., R. R. White, R. M. Senior, R. J. Rodriguez, and C. Kuhn. 1979. Receptor mediated binding and internalization of leukocyte and elastase by alveolar macrophages in vitro. J. Clin. Invest. 64:824-833.

33. McGowan, S. E., R. D. Arbeit, P. J. Stone, and G. L. Snider. 1983. A comparison of the binding and fate of internalized neutrophil elastase in human monocytes and alveolar macrophages. Am. Rev. Respir. Dis. 128:688-694.

34. Chin, J. R., G. Murphy, and Z. Werb. 1985. Stromelysin, a connective tissue-degrading metalloendopeptidase secreted by stimulated rabbit synovial fibroblasts in parallel with collagenase. J. Biol. Chem. 260:12367-12376.

35. Hasty, K. A., M. S. Hibbs, A. H. Kang, and C. L. Mainardi. 1986. Secreted forms of neutrophil collagenase. J. Biol. Chem. 261:5645-5650.

36. Murphy, G., C. G. McAlpine, C. T. Poll, and J. J. Reynolds. 1985. Purification and characterization of a bone metalloproteinase that degrades gelatin and types IV and V collagens. Biochim. Biophys. Acta. 831:49-58.

37. Banda, M. J., and Z. Werb. 1981. Mouse macrophage elastase. Biochem. J. 193:589-605.

38. Louie, J. S., J. Weiss, L. Ryhanan, K. M. Nies, S. Rantala-Ry- hanen, and J. Uitto. 1984. The production of collagenase by adherent mononuclear cells cultured from human blood. Arthritis Rheum. 27:1397-1404.

39. Gadek, J., G. Hunninghake, R. Zimmerman, and R. Crystal. 1981. Production of connective tissue specific proteases by human alveolar macrophages is constitiutive (non-regulatable). Am. Rev. $R e$ spir. Dis. 128:55. (Abstr.)

40. Bar-Shavit, Z., S. L. Teitelbaum, G. P. Striklin, A. Z. Eisen, A. J. Kahn, and H. G. Welgus. 1985. Differentiation of a human leukemia cell line and expression of collagenase inhibitor. Proc. Natl. Acad. Sci. USA. 82:5380-5384.

41. Welgus, H. G., N. L. Connolly, and R. M. Senior. 1986. 12-0Tetradecanoyl-phorbol-13-acetate differentiated U937 cells express a macrophage-like profile of neutral proteinases. High levels of secreted collagenase and collagenase inhibitor accompany low levels of intracellular elastase and cathepsin G. J. Clin. Invest. 77:1675-1681.

42. Hasty, K. A., M. S. Hibbs, A. H. Kang, and C. L. Mainardi. 1985. Heterogeneity among human collagenase demonstrated by a monoclonal antibody that selectively recognizes and inhibits human neutrophil collagenase. J. Exp. Med. 159:1455-1463.

43. Hasty, K. A., J. M. Jeffrey, M. S. Hibbs, and H. G. Welgus. 1986. Comparative kinetic behavior of human neutrophil and human skin fibroblast collagenase. J. Biol. Chem. 262:10048-10052.

44. Hibbs, M. S., and A. H. Kang. 1986. Expression of metalloproteinases which degrade gelatin and native type $\mathrm{V}$ collagen by resident and inflammatory cells. J. Cell. Biochem. 10A:262. (Abstr.)

45. Garbisa, S., M. Ballin, D. Daga-Gordini, G. Fastelli, M. Naturale, A. Negro, G. Semenzato, and L. Liotta. 1986. Transient expression of type IV collagenolytic metalloproteinase by human mononuclear phagocytes. J. Biol. Chem. 261:2369-2375. 